\title{
Gestión de la bioseguridad odontológica y el riesgo de contagio por covid-19 del cirujano dentista de la comisión de salud bucal de Lima, 2021
}

\author{
Mgtr. Nancy Mariely Ulloa Ale \\ nancy.ulloa@unmsm.edu.pe \\ https://orcid.org/0000-0002-6161-3447 \\ Mgtr. Claudia Alejandra Cavero Chavez \\ claudia_1312_4@hotmail.com \\ https://orcid.org/0000-0003-4117-1079 \\ Universidad César Vallejo \\ Lima - Perú
}

\section{RESUMEN}

La presente investigación tuvo como objetivo determinar la relación entre la gestión de la bioseguridad odontológica y el riesgo de contagio por COVID- 19 del cirujano dentista de la comisión de salud bucal de Lima, 2021. El estudio tuvo un enfoque cuantitativo, tipo básico, el método hipotético deductivo, de nivel explicativo y de diseño no experimental, correlacional. La muestra fue de 110 cirujanos dentistas. Aplicándose la encuesta como técnica y el cuestionario como instrumento para cada variable; que fueron previamente validados por expertos. Donde se obtuvo como resultado que un $62,7 \%$ afirma tener una adecuada gestión de la bioseguridad y un 61,8 \% bajo riesgo de contagio por COVID-19 y según la prueba de contraste de hipótesis $(\mathrm{p}=0,000$; rho de Spearman = 0,978). Concluyéndose, que entre la gestión de la bioseguridad odontológica y el riesgo de contagio por COVID-19 del cirujano dentista de la comisión de salud bucal de Lima,2021; existe una relación significativa y presenta una correlación positiva muy fuerte.

Palabras clave: gestión; bioseguridad; odontológica; riesgo de contagio; covid- 19 


\title{
Management of dental biosafety and the risk of contagion by covid-19 of the dental surgeon of the oral health commission of Lima, 2021
}

\begin{abstract}
The objective of this research was to determine the relationship between dental biosafety management and the risk of contagion by COVID-19 of the dental surgeon of the Oral Health Commission of Lima, 2021. The study had a quantitative approach, basic type, the hypothetical deductive method, explanatory level and non-experimental, correlational design. The sample consisted of 110 dental surgeons. Applying the survey as a technique and the questionnaire as an instrument for each variable; that were previously validated by experts. Where it was obtained as a result that $62.7 \%$ claim to have adequate biosafety management and $61.8 \%$ low risk of contagion by COVID-19 and according to the hypothesis contrast test $(\mathrm{p}=0.000$; Spearman's rho $=0.978)$. Concluding, that between the management of dental biosafety and the risk of contagion by COVID-19 of the dental surgeon of the oral health commission of Lima, 2021; there is a significant relationship and presents a very strong positive correlation.
\end{abstract}

Keywords: management; biosecurity; dental; risk of contagion; covid- 19

Artículo recibido: 30 noviembre. 2021 Aceptado para publicación: 29 diciembre 2021 Correspondencia: nancy.ulloa@unmsm.edu.pe Conflictos de Interés: Ninguna que declarar 


\section{INTRODUCCIÓN}

El coronavirus SARS-COV-2 inicio su propagación en Wuhan- China el 2019, transmitiéndose por tos y estornudos; ocasionando una pandemia y crisis sanitaria; exigiendo a los sistemas públicos de salud y personal de salud en todo el mundo incrementar sus atenciones según Ningthoujam (2020), Mahase (2020) y WHO, (2020). En China el 3, 8\% del personal de salud registro la infección del virus, en cambio los salubristas italianos resultaron el $20 \%$ infectados (Remuzzi, A. y Remuzzi, G., 2020). Siendo el 5\% del total muertes de dentistas a nivel mundial (Meng et al., 2020). En diversos países adoptaron medidas para restringir la movilidad humana y evitar la propagación, lo mencionaron Andersen et al. (2020) y Kraemer et al. (2020).

Por lo que internacionalmente se publicaron protocolos clínicos innovados de gestión de bioseguridad, control de tratamiento y tiempo asignado por paciente en pro de preservar la salud del personal de salud y pacientes; OMS (2020, Barabari y Moharamzadeh, (2020) y Sigua-Rodríguez et al. (2020).

En América Latina hubo una morbilidad y mortalidad en la población de 26,967,312 y 881,096 respectivamente por COVID-19 (Universidad de Johns Hopkins [UJH], 2020). Así mismo el Ministerio de Salud (MINSA) reportó en el Perú datos estadísticos, hasta el mes de agosto del 2020, siendo los casos positivos en la población de 702,776 y de letalidad 30, 236 (MINSA, 2020).

Así también, el MINSA (2020), propuso como protocolo de gestión de la Bioseguridad en odontología, procedimientos para atender a los pacientes, cuidados para los salubristas, manejo correcto del material e instrumental, manejo del ambiente odontológico, uso de barreras protectoras, manejo de residuos contaminados y medidas básicas frente a accidentes de exposición a sangre o fluidos corporales. Según la Asociación dental Americana (2020) y Meng, Hua y Bian (2020); en diversos países se emitieron protocolos internacionales de gestión de bioseguridad, así también Vera (2020), en el Colegio de Odontólogos del Perú (COP), recomendo que durante la emergencia sanitaria solo atendieran emergencias o urgencias dentales $y$ recomendaron al gremio las inmunizaciones.

Todos los cirujanos dentistas pueden contraer diversas patologías ejerciendo su carrera, ya que trabajan en la cavidad bucal estando expuestos a fluidos corporales, por ende, es imprescindible evaluar el riesgo de contagio por COVID-19. Deacuerdo a los signos y 
síntomas presentes puede ser un caso sospechoso, si estado cerca alguien que presenta la enfermedad se convierte en un caso probable y si se realizado un examen de laboratorio y salió positivo a COVID-19 es un caso confirmado. Deacuerdo a la exposición de las personas al virus presentan un riesgo alto, medio o bajo a esta enfermedad.

Con respecto a los dentistas peruanos según Zambrano (2021), al inicio de la pandemia se evidencio 157 casos de contagio de COVID-19 y 16 defunciones (10\%), sin embargo, en octubre hubo 348 casos de contagio con una letalidad de 44 (11\%); teniendo un $70 \%$ de contagios la región costa. Dentro de las especialidades los más afectados fueron los periodoncistas $(32,2 \%)$ y el tratamiento de preparación dentaria evidencio un mayor riesgo de contagio $(69,4 \%)$ según Benli et al. (2021).

Los integrantes de comisión de salud bucal están conformados por 121 dentistas de Lima perteneciente al sector privado, por lo cual tuvieron que gestionar la bioseguridad, adquiriendo ellos mismos sus EPP, instrumentales, materiales e insumos, a pesar de la escasez y alto presupuesto; así como la inmunización ya que en un inicio hubo una inadecuada gestión entre el COP, MINSA y dentistas; incorporando barreras sanitarias en los ambientes de trabajo y desechos biocontaminados. Al inicio de la pandemia se dieron múltiples teorías sobre la forma de los contagios ya que no se sabía con precisión los signos y síntomas patognomónicos de la enfermedad creando una incertidumbre y riesgo de contagio por COVID -19 en los dentistas.

Por lo que el problema general planteado es: ¿Cómo se relaciona la gestión de la bioseguridad odontológica y el riesgo de contagio por COVID-19 del cirujano dentista de la comisión de salud bucal de Lima, 2021?, y como problemas específicos: (i)¿Cómo se relaciona el cuidado del personal odontológico y el riesgo de contagio por COVID-19 del cirujano dentista de la comisión de salud bucal de Lima, 2021?; (ii) ¿Cómo se relaciona el manejo del material e instrumental y el riesgo de contagio por COVID-19 del cirujano dentista del cirujano dentista de la comisión de salud bucal de Lima, 2021?; y (iii) ¿Cómo se relaciona el ambiente odontológico y el riesgo de contagio por COVID19 del cirujano dentista del cirujano dentista de la comisión de salud bucal de Lima, 2021 .

La justificación que se utilizó para este estudio de investigación fue: (a) La justificación teórica, se consideró necesario propuestas para contribuir a mejorar la Gestión de la bioseguridad odontológica y riesgo de contagio por COVID-19 del cirujano dentista en 
el distrito de Lima, 2021 y permitirnos conocer la aplicación de la normatividad y legalidad que establece el Ministerio de Salud y tomar esta información como base para futuros estudios e incrementar los conocimientos en la comunidad científica, educativa, (b) Con relación a la justificación metodológica fue descriptiva, cuantitativo, correlacional, empleando un instrumento como el cuestionario y las técnicas estadísticas de tipo descriptivo para diagnosticar sobre la gestión de la bioseguridad odontológica y riesgo de contagio por COVID-19 del cirujano dentista en el distrito de Lima, y a nivel inferencial para obtener conclusiones a partir de una muestra de estudio. Además, se validó y adaptó un instrumento para obtener información del presente estudio; la validación del constructo de instrumento constó de 34 ítems. (c) Respecto a la justificación práctica, es prescindible conocer sobre la bioseguridad frente al COVID-19 y poder proponer un plan de trabajo e implementación de infraestructura e insumos que preserven la salud del cirujano dentista, eviten la contaminación cruzada, lo que beneficiará la atención del paciente, mediante una implementación de protocolos de bioseguridad para disminuir el riesgo de contagio por COVID- 19.

En relación a objetivos, el general fue: Determinar la relación entre la gestión de la bioseguridad odontológica y el riesgo de contagio por COVID- 19 del cirujano dentista de la comisión de salud bucal de Lima; y específicos fueron: (I) Determinar la relación del cuidado personal odontológico y el riesgo de contagio por COVID-19 del cirujano dentista de la comisión de salud bucal de Lima, 2021; (II) Determinar la relación del manejo del material e instrumental y el riesgo de contagio por COVID-19 del cirujano dentista de la comisión de salud bucal de Lima, 2021; (III) Determinar la relación del ambiente odontológico y el riesgo de contagio por COVID-19 del cirujano dentista de la comisión de salud bucal de Lima, 2021. Formulándose la hipótesis general: La gestión de la bioseguridad odontológica se relaciona con el riesgo de contagio por COVID- 19 del cirujano dentista de la comisión de salud bucal de Lima, 2021; al igual que las siguientes hipótesis específicas: (i) El cuidado del personal odontológico se relaciona con el riesgo de contagio por COVID-19 del cirujano dentista de la comisión de salud bucal de Lima, 2021; (ii) El manejo del material e instrumental se relaciona con el riesgo de contagio por COVID-19 del cirujano dentista de la comisión de salud bucal de Lima, 2021; y (iii) El ambiente odontológico se relaciona con el riesgo de contagio por COVID- 19 del cirujano dentista de la comisión de salud bucal de Lima, 2021. 


\section{ESTRATEGIAS METODOLÓGICAS O MATERIALES Y MÉTODOS}

\section{Tipo de estudio}

El paradigma del estudio fue positivista, porque tuvo como finalidad verificar una hipótesis por procedimientos estadísticos o establecer los parámetros de una variable mediante la expresión numérica (Ramos, 2017). El enfoque fue cuantitativo, para medir los datos de las variables estudiadas por datos numéricos, comparando y realizando el contraste de hipótesis (Sánchez et al., 2018). Así también fue de tipo básica o sustantiva ya que busca nuevos conocimientos, que sirven para realizar una investigación aplicada. Y, se aplicó el método hipotético deductivo el cual ayuda a las predicciones para luego ser sometidas al contraste o prueba llegando a establecer las conclusiones del estudio (Pérez y Rodríguez, 2017)..

\section{Diseño}

El diseño fue no experimental, porque se observó las variables gestión de bioseguridad y riesgo de contagio. Así también tuvo una correlacional causal y estableció la relación de las variables mediante su causa y efecto. Describió

Sánchez et al. (2018), como la relación, incidencia mediante el cual se puede inferir las posibles relaciones posteriores causales. La investigación tuvo nivel explicativo, creando y elaborando teorías y añadiendo predicciones a los fundamentos científicos mediante la significancia (Abreu, 2012). La investigación busco saber porque de los resultados mediante el método sintético y analítico según Hernández y Mendoza (2018).

Figura 1. Diagrama del diseño correlacional

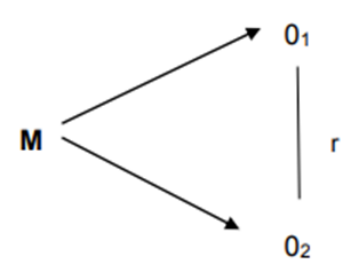

\section{Dónde:}

$\mathrm{M}=$ Muestra

O1= Observación de la V1

$\mathrm{O} 2=$ Observación de la V2

$\mathrm{r}=$ Correlación entre las variables 


\section{Población y muestra}

\section{Población}

La población estuvo conformada por 110 cirujanos dentistas que pertenecen a la comisión de salud bucal región Lima.

\section{Muestra}

En el estudio se realizó una muestra tipo censal, trabajándose con la población en su totalidad; integrada por 110 cirujanos dentistas que pertenecen a la comisión de salud bucal región Lima.

\section{Técnicas e instrumentos de recolección de datos}

Técnicas: La técnica de recolección de datos que se utilizó en esta indagación ha sido la encuesta. Esta técnica se resume en un formato que se aplica a una muestra de informantes, para obtener datos (Arroyo, 2020).

Instrumento: para la investigación se empleó cuestionario, cual consta de 37 preguntas de la Escala Likert.

\section{Validación y confiabilidad del instrumento (s)}

La validez y confiabilidad, del cuestionario ha sido sometido a métodos para implementar su validez y confiabilidad. La validez ha sido definida por el criterio de 03 jueces y por medio del estudio de ítems. La variable gestión de bioseguridad odontológica tuvo un alfa de Cronbach de 0,789 y la variable riesgo de contagio COVID-19 obteniendo un alfa de Cronbach 0,801.

\section{RESULTADOS Y DISCUSIÓN}

Tabla 1

Distribución de los niveles de las variables gestión de bioseguridad y riesgo de contagio por COVID-19 del cirujano dentista de la comisión de salud bucal de Lima, 2021

\begin{tabular}{|c|c|c|c|c|c|c|c|}
\hline & & \multicolumn{4}{|c|}{ Riesgo de contagio por COVID-19 } & \multicolumn{2}{|c|}{ Total } \\
\hline & & \multicolumn{2}{|c|}{ Nivel bajo } & \multicolumn{2}{|c|}{ Nivel medio } & & \\
\hline & & Cant. & $\%$ & Cant. & $\%$ & \multicolumn{2}{|c|}{ Cant. } \\
\hline \multirow{3}{*}{$\begin{array}{l}\text { Gestión } \\
\text { Bioseguridad } \\
\text { Odontológica }\end{array}$} & Nivel bajo & 0 & & 1 & $0,9 \%$ & 1 & $0,9 \%$ \\
\hline & Nivel medio & 0 & & 40 & $36,4 \%$ & 40 & $36,4 \%$ \\
\hline & Nivel alto & 68 & $61,8 \%$ & 1 & $0,9 \%$ & 69 & $62,7 \%$ \\
\hline Total & & 68 & 61,8 & 42 & 38,2 & 110 & $100 \%$ \\
\hline
\end{tabular}




\section{Tabla 2}

Distribución de niveles de las dimensiones de la variable gestión de la bioseguridad odontológica del cirujano dentista de la comisión de salud bucal de Lima, 2021

\begin{tabular}{lcccccc}
\hline Niveles & \multicolumn{2}{c}{$\begin{array}{c}\text { Cuidado del personal } \\
\text { odontológico }\end{array}$} & \multicolumn{2}{c}{$\begin{array}{c}\text { Manejo de material e } \\
\text { instrumental }\end{array}$} & \multicolumn{2}{c}{$\begin{array}{c}\text { Ambiente } \\
\text { odontológico }\end{array}$} \\
\hline & $\mathbf{f}$ & $\mathbf{\%}$ & $\mathbf{f}$ & $\mathbf{\%}$ & $\mathbf{f}$ & $\%$ \\
Bajo & 0 & $0 \%$ & 7 & $6,4 \%$ & & \\
Medio & 34 & $30,9 \%$ & 15 & $13,6 \%$ & 47 & $42,7 \%$ \\
Alto & 76 & $69,1 \%$ & 88 & $80,0 \%$ & 63 & $57,3 \%$ \\
\hline Total & $\mathbf{1 1 0}$ & $\mathbf{1 0 0 \%}$ & $\mathbf{1 1 0}$ & $\mathbf{1 0 0 \%}$ & $\mathbf{1 1 0}$ & $\mathbf{1 0 0 \%}$ \\
\hline
\end{tabular}

\section{Tabla 3}

Distribución de niveles de las dimensiones de la variable riesgo de contagio COVID-19 del cirujano dentista de la comisión de salud bucal de Lima, 2021

\begin{tabular}{lccccccc}
\hline & Nivel & \multicolumn{2}{c}{ Caso Sospechoso } & \multicolumn{2}{c}{ Caso Probable } & \multicolumn{2}{c}{$\begin{array}{c}\text { Caso } \\
\text { Confirmado }\end{array}$} \\
\hline & F & \% & F & $\%$ & F & $\%$ \\
Bajo & 77 & 70 & 66 & 60 & 66 & 60 \\
$\begin{array}{l}\text { Medio } \\
\text { Alto }\end{array}$ & 33 & 30 & 44 & 40 & 44 & 40 \\
\hline \multicolumn{1}{c}{ Total } & $\mathbf{1 1 0}$ & $\mathbf{1 0 0}$ & $\mathbf{1 1 0}$ & $\mathbf{1 0 0}$ & $\mathbf{1 1 0}$ & $\mathbf{1 0 0}$ \\
\hline
\end{tabular}

\section{Tabla 4}

Correlación de las variables gestión de bioseguridad y riesgo de contagio por COVID-19 del cirujano dentista de la comisión de salud bucal de Lima, 2021

\begin{tabular}{|c|c|c|c|c|}
\hline & Vari & & $\begin{array}{c}\text { Gestión de } \\
\text { bioseguridad }\end{array}$ & $\begin{array}{c}\text { Riesgo de } \\
\text { contagio }\end{array}$ \\
\hline & Gestión de & $\begin{array}{l}\text { Coeficiente de } \\
\text { correlación }\end{array}$ & 1,000 & 0,978 \\
\hline & bioseguridad & Sig. (bilateral) & . & ,000 \\
\hline Rho de & & $\mathrm{N}$ & 110 & 110 \\
\hline Spearman & Riesgo de & $\begin{array}{l}\text { Coeficiente de } \\
\text { correlación }\end{array}$ & 0,978 & 1,000 \\
\hline & contagio & Sig. (bilateral) & ,000 & . \\
\hline & & $\mathrm{N}$ & 110 & 110 \\
\hline
\end{tabular}




\section{Tabla 5}

Correlación de la dimensión cuidado del personal odontológico y la variable riesgo de contagio por COVID-19 del cirujano dentista de la comisión de salud bucal de Lima, 2021

\begin{tabular}{|c|c|c|c|c|}
\hline & Variable & & $\begin{array}{c}\text { Cuidado del personal } \\
\text { odontológico }\end{array}$ & $\begin{array}{c}\text { Riesgo de } \\
\text { contagio }\end{array}$ \\
\hline \multirow{6}{*}{$\begin{array}{l}\text { Rho de } \\
\text { Spearman }\end{array}$} & \multirow{3}{*}{$\begin{array}{l}\text { Cuidado del } \\
\text { personal } \\
\text { odontológico }\end{array}$} & $\begin{array}{l}\text { Coeficiente } \\
\text { correlación }\end{array}$ & 1,000 & 0,851 \\
\hline & & Sig. (bilateral) & & ,000 \\
\hline & & $\mathrm{N}$ & 110 & 110 \\
\hline & \multirow{3}{*}{$\begin{array}{l}\text { Riesgo de } \\
\text { contagio }\end{array}$} & $\begin{array}{l}\text { Coeficiente } \\
\text { correlación }\end{array}$ & 0,851 & 1,000 \\
\hline & & Sig. (bilateral) & ,000 & . \\
\hline & & $\mathrm{N}$ & 110 & 110 \\
\hline
\end{tabular}

\section{Tabla 6}

Correlación de la dimensión manejo del material e instrumental y la variable riesgo de contagio por COVID-19 del cirujano dentista de la comisión de salud bucal de Lima, 2021

\begin{tabular}{|c|c|c|c|c|c|}
\hline \multicolumn{3}{|c|}{ Variable } & & $\begin{array}{l}\text { Manejo de material e } \\
\text { instrumental }\end{array}$ & $\begin{array}{l}\text { Riesgo de } \\
\text { contagio }\end{array}$ \\
\hline \multirow{6}{*}{$\begin{array}{l}\text { Rho de } \\
\text { Spearman }\end{array}$} & \multirow{3}{*}{$\begin{array}{l}\text { Manejo de } \\
\text { material e } \\
\text { instrumental }\end{array}$} & $\begin{array}{l}\text { Coeficiente } \\
\text { correlación }\end{array}$ & de & 1,000 & 0,633 \\
\hline & & Sig. (bilateral) & & &, 000 \\
\hline & & $\mathrm{N}$ & & 110 & 110 \\
\hline & \multirow{3}{*}{$\begin{array}{l}\text { Riesgo de } \\
\text { contagio }\end{array}$} & $\begin{array}{l}\text { Coeficiente } \\
\text { correlación }\end{array}$ & de & 0,633 & 1,000 \\
\hline & & Sig. (bilateral) & & ,000 & . \\
\hline & & $\mathrm{N}$ & & 110 & 110 \\
\hline
\end{tabular}

\section{Tabla 7}

Correlación de la dimensión ambiente odontológico y la variable riesgo de contagio por COVID-19 del cirujano dentista de la comisión de salud bucal de Lima, 2021

\begin{tabular}{lllcc}
\hline \multicolumn{2}{c}{ Variable } & $\begin{array}{c}\text { Ambiente } \\
\text { odontológico }\end{array}$ & $\begin{array}{c}\text { Riesgo de } \\
\text { contagio }\end{array}$ \\
\hline & $\begin{array}{l}\text { Ambiente } \\
\text { odontológico }\end{array}$ & Coeficiente de correlación & 1,000 & 0,910 \\
& & Sig. (bilateral) & $\cdot$ &, 000 \\
Rho de Spearman & & Coeficiente de correlación & 0,910 & 1,000 \\
& \multirow{2}{*}{$\begin{array}{l}\text { Riesgo de } \\
\text { contagio }\end{array}$} & Sig. (bilateral) &, 000 & $\cdot$ \\
& & N & 110 & 110 \\
\hline
\end{tabular}


En la tabla 1, visualizamos los niveles de gestión de bioseguridad odontológica obteniéndose un nivel bajo con un $0,9 \%$, medio con un $36,4 \%$ y siendo el de mayor porcentaje un nivel alto con $62,7 \%$. Con respecto a la variable riesgo de contagio COVID19 se evidenciaron los niveles de mayor porcentaje bajo, con un $61,8 \%$, seguido por el nivel medio con un $38,2 \%$.

Además, en la tabla 2, se presentó los niveles de la dimensión cuidado del personal odontológico obteniendo de resultado que ningún odontólogo evidencia un nivel bajo, que $34(30,9 \%)$ odontólogos presentaron un nivel medio y $76(69,1 \%)$ un nivel alto. Además, en el nivel de la dimensión manejo de material e instrumental se pudo apreciar que un $7(6,4 \%)$ tiene un nivel bajo, mientras que $15(13,6 \%)$ un nivel medio y $88(80 \%)$ un nivel alto. De la misma manera en el nivel de la dimensión ambiente odontológico dio como resultado que ningún odontólogo tiene un nivel bajo, mientras que 47 (42,7\%) un nivel medio y $63(57,3 \%)$ un nivel alto.

Luego, en la tabla 3, se presenta los niveles de la dimensión caso sospechoso obteniendo de resultado que 77 (70\%) odontólogos presentaron un nivel bajo, 33(30\%) un nivel medio y ningún odontólogo evidencia un nivel alto. Además, en el nivel de la dimensión caso probable se pudo apreciar que un $66(60 \%)$ tiene un nivel bajo, mientras que 44 $(40 \%)$ un nivel medio y ninguno un nivel alto. De la misma manera en el nivel de la dimensión caso confirmado obtuvimos que 66 (60\%) tiene un nivel bajo, mientras que 44 (40\%) un nivel medio y ningún odontólogo tuvo un nivel alto.

En la tabla 4, evidenciamos la matriz de correlación de las variables gestión de bioseguridad y riesgo de contagio por COVID-19, por lo que se puede evidenciar que según el resultado obtenido existe una relación significativa y presenta una correlación positiva muy fuerte, $(\mathrm{p}=0,000$; rho de Spearman $=0,978)$. De esta manera se comprueba lo expresado por la teoría de organización o de Fayol que en una organización debe destacar la eficiencia y planeación para un adecuado bienestar, y seguridad de la organización y de los trabajadores, (Almanza et al.; 2018). De la misma forma la teoría del principio básico de bioseguridad indica como característica la universalidad, ya que todas las personas pueden portar alguna patología; por ello se debe utilizar adecuadamente el uso de equipo de protección personal y el método de eliminar materia contaminada adecuadamente (Cuba, 2021) para evitarla infección cruzada. Así mismo, la Triada Ecológica (Cohen y Shang, 2015), indica que los huéspedes, los agentes y el 
medio ambiente relacionados entre ellos, pueden iniciar una etiopatogenia, por lo cual se recomienda el uso de EPP en los huéspedes (cirujanos dentistas) para disminuir el riesgo de contagio en el centro laboral. Además, lo propuesto por la teoría general de evaluación de riesgo, menciona la importancia de adecuada gestión de bioseguridad para preservar la salud y el riesgo de contagio en sus trabajadores; que es complementado por lo que menciona CIGET (2020), sobre una constante evaluación con respecto al riesgo en los centros dentales por superintendencias en salud. Con respecto al trabajo realizado, los siguientes autores corroboran la información obtenida: Barbosa-Liz et al. (2021), indica que el uso de elementos de protección personal fue mayor al 99\% en odontólogos y el autoreporte de contagio por COVID-19 fue bajo $(0,61 \%)$. Concluyendo que el seguimiento de protocolos de bioseguridad y de uso de EPP fue alto mientras el contagio autoreportado fue muy bajo. Así también Estrich (2020), manifiesta de que solo el 0,9\% tenía COVID-19 confirmado o probable; indicando que las recomendaciones de control de infecciones, gestión de bioseguridad actuales ayudan prevenir infecciones en entornos dentales. Luego Putrino et. al. (2020), evidenciaron un nivel alto de información lo cual favoreció a los cirujanos dentistas a mejorar las medidas de gestión de bioseguridad frente a la propagación del virus. Además, COVIDental Collaboration Group (2021), concluyo que la profesión dental es de alto riesgo, sin embargo, las tasas de COVID-19 informadas para los profesionales dentales fue solo de 3,19 (\%) por lo que no fueron significativamente diferentes de la población general. Luego Marchena (2021), indico que si se relaciona gestión de la bioseguridad odontológica con riesgo de contagio (RC) por COVID-19; respecto a gestión de bioseguridad odontológica presento un nivel alto con $59,5 \%$, mientras que un nivel de bajo riesgo de contagio $90,9 \%$.

En la tabla 5, evidenciamos la matriz de correlación de la dimensión cuidado del personal odontológico y la variable riesgo de contagio por COVID-19, donde se puede evidenciar que según el resultado obtenido en la contrastación, existe una relación significativa y tiene una correlación positiva considerable, $(\mathrm{p}=0,000$; rho de Spearman $=0,851)$, presentando el 69,1\% un nivel alto de cuidado personal odontológico. Es así que los resultados encontrados enaltecen la temática de la teoría General del Autocuidado de Dorothea (1969) que consideran favorecedores un adecuado auto cuidado en la persona para la preservación de su salud. Por estas razones, es prescindible una máxima bioseguridad en las atenciones odontológicas frente al COVID-19 (Naranjo-Hernández 
et al. 2017). De la misma forma incide en la teoría del Cuidado Humano, en cuanto al profesional tiene que estar comprometido en proteger, mejorar y preservar la salud del paciente; respetando el código deodontología y ética Watson (1979, citado por GuerreroRamírez et al., 2017). Así mismo, dichos resultados encontrados son reafirmados por los resultados de Barbosa-Liz et al. (2021) ya que el 99\% de odontólogos siguen los protocolos de bioseguridad y de uso de EPP fue alto, aunque la percepción de riesgo de contagio fue alta, el contagio autoreportado fue muy bajo. Además, concuerda con lo indicado por Benli et al. (2021), que los odontólogos realizan una adecuada gestión de bioseguridad acorde con la especialidad e instrumentos que utilizan, evaluando los de riesgo de contagio de COVID-19 sin embargo tenían un conocimiento limitado sobre la viabilidad del virus. Luego Miguelena et al. (2021), en un estudio descriptivo y transversal, encuestaron a 200 dentistas privados a través de redes sociales teniendo como resultado que $16.5 \%$ ha padecido COVID-19, reconociendo que han tenido que reforzar el uso de EPP y algunos protocolos de protección. Concluyendo que la profesión representa un alto riesgo de contagio y la posibilidad de generar una infección cruzada por el virus SARS-CoV-2, por lo que se tiene que mejorar la gestión de bioseguridad y ser parte el plan de vacunación como parte importante del sector salud (AU). Así mismo Alava-Zambrano et al. (2021) en su estudio seroprevalencia factores de riesgo y los protocolos de bioseguridad en 128 profesionales de salud. encontraron que la seroprevalencia fue de 22,65\% (enero 2021- SARS-CoV-2), concluyeron que los profesionales de salud utilizaron adecuadamente los protocolos de bioseguridad. De tal manera Estrich (2020), encuestó a 2195 dentistas privados y públicos de EE. UU. sobre la prevalencia de COVID-19 y las tasas de positividad. Donde manifestaron tener una adecuada gestión de bioseguridad ya que el 99,6\% de los dentistas indicaron el uso de EPP al tratar a los pacientes, en el 99,7\% de las prácticas incluyeron desinfección de superficies, distanciamiento social y uso de cubiertas faciales. prevenir infecciones en entornos dentales y reduciendo el riesgo de contagio COVID-19. Finalmente, Wong et al. (2020) y Coulthard (2020), el uso de mascarillas de alta calidad parece ser la medida eficaz para evitar la infección por SARS-CoV-2. Desde el inicio del VIH / SIDA y la mayor conciencia sobre la hepatitis B y C en los años 1990, la profesión dental adoptó estrictas medidas de control de infecciones. El centro de control de Enfermedades (CDC) publicó directrices para el control de infecciones partiendo de la suposición de que todos 
los pacientes de un consultorio dental podrían estar infectados con el VIH o los virus de la hepatitis B.

En la tabla 6, podemos ver la correlación de la dimensión manejo del material e instrumental y la variable riesgo de contagio por COVID-19, se puede evidenciar que según el resultado obtenido en la contrastación existe una correlación positiva media, $(\mathrm{p}=0,000$; rho de Spearman $=0,633)$, de la misma forma el $80 \%$ manifestó tener un nivel alto de manejo de material e instrumental. Corroborando lo que expresa la teoría de las relaciones humanas donde el ser humano desarrolla conocimientos, destrezas y habilidades individuales (Segredo, 2017), por lo cual es necesario una adecuada destreza en las funciones realizadas por los dentistas en el manejo del instrumental para reducir el riesgo de contagio. Por lo cual en el modelo explicativo de la teoría del germen a la de los factores de riesgo: enfermedades infecciosas, agentes patógenos específicos producían enfermedades (Barret-Connor, 1998, citado por Urquía, 2019). Recomendando un adecuado protocolo de desinfección, limpieza y esterilización para evitar el riesgo de contagio. Ratificado por Mendoza, P. y Mendoza, D. (2020), en una revisión sobre el riesgo de transmisión y bioseguridad del SARS-Cov-2 indico el alto riesgo de contagio por aerosoles ya que el virus es detectable en estos hasta por tres horas y son producidos por instrumentales, y durante este tiempo los odontólogos estar expuestos a ellas; especialmente cuando son atendidos los pacientes asintomáticos. Es prescindible conocer el adecuado manejo de material e instrumental para una adecuada esterilización, así como las características del riesgo de transmisión y medidas de prevención 1 a fin de bloquear la probabilidad de contagiarse. Es preciso señalar que Menjivar et al. (2021), investigaron el cumplimiento de desinfección y esterilización del instrumental que se utiliza en las Unidades de Salud del Área Metropolitana de San Salvador encontrando que el $94.3 \%$ realiza el lavado del instrumental odontológico se aprecia el $97.1 \%$ desinfectan el instrumental $100 \%$ esteriliza el instrumental odontológico y de ellos el $91.4 \%$ esteriliza el instrumental odontológico con calor seco, el $5.7 \%$ esteriliza con calor húmedo y el $2.9 \%$ utilizan alternadamente la esterilización. Concluyendo que cumplen las medidas adecuadas de desinfección y esterilización. Igualmente, Cagetti et al. (2020), con el análisis de indagación evaluaron medidas de precauciones en cirujanos dentistas donde el $86,97 \%$ de ellos procuraban tiempo considerable en la sanitización de los equipos e instrumentales de trabajo , contando el 88,98\% con una amplia ventilación en la sala para 
esperar, mientras el 91,64\% manifestaron lavarse las manos anteriormente y luego de las atenciones .Concluyendo que los odontólogos dan una gran importancia al adecuado manejo de instrumental odontológico y el proceso de lavado de manos.

En la tabla 7, Correlación de la dimensión ambiente odontológico y la variable riesgo de contagio por COVID-19, se puede evidenciar que según el resultado obtenido en la contrastación existe una correlación positiva muy fuerte, $(\mathrm{p}=0,000$; rho de Spearman $=$ 0,910). Se corrobora con la teoría de Florencia Nightingale o del entorno que enfatizó la importancia de la asepsia y de mantener los ambientes limpios (Urquía, 2019). Así también la teoría de sistemas sociales de la organización indica que hay que adaptarse a un entorno de constantemente cambio, con el fin de lograr objetivos implicando en las organizaciones (Kreps ,1996). Además, las Teorías Microbianas de patologías infectocontagiosas, indican su propagación de personas infectadas a sanas. Ya que, el virus COVID-19 presenta una gran y rápida propagación contagiosa respiratoria (WHO, 2020). De la misma forma la teoría de multicausalidad, indica la patogenia a diversos factores pudiendo ser intrínsecos (inmunidad) o extrínsecos (biológicos, sociales, etc.) durante su ciclo vital (Arredondo, 1992). De los antecedentes descritos mencionaremos a quienes corroboran con los resultados de la investigación como lo son Cari y Zúñiga (2017), que utilizó un cuestionario de 25 preguntas diseñadas de acuerdo a la normatividad del ministerio de salud, estudio es descriptivo explicativo, transversal y prospectivo. El 78,7\% de estudiantes y personal de clínica cumplen con la norma técnica del Ministerio de Salud, el 56\% de estudiantes y personal cumple con la norma técnica se adecúa de acuerdo a la norma técnica respecto al acondicionamiento, segregación, almacenamiento primario y almacenamiento intermedio concluyendo que hay un adecuado cuidado del ambiente odontológico. Además, Gómez y Prieto (2021), indicaron el alto riesgo de contagio ante el SARS-CoV-2 en la odontología, Por lo que se debe tomar medidas de protección y agendar consultas mixtas (presenciales y telefónicas), para descartar pacientes con sintomatología, actualizándose sobre vías de transmisión y medidas para su prevención en el ámbito odontológico. Concluyendo que se debe instaurar las medidas de gestión de bioseguridad que minimicen el riesgo de contagio tanto para los pacientes como para los profesionales. Así también Aquino-Canchari (2020), evidenció que el SARS-CoV-2 puede alcanzar hasta 1,82 metros en la saliva del paciente, estando expuestos a gran variedad de microorganismos y posibles contagios, 
por lo que odontólogos y profesionales de salud deben tener una buena gestión de bioseguridad cuidando el ambiente donde laboran. Además, indicó que hay desinformación con respecto al Virus COVID-19, ya que su riesgo de letalidad es ínfimo comparado al SIDA, hepatitis, ébola etc. Tal cual Alba et al. (2020), evaluaron el cumplimiento de los protocolos del manual para la gestión integral de los residuos donde el $89,10 \%$ de los participantes manifiesta que deposita de forma correcta los residuos sólidos; el 83,97 \% considera que clasifica correctamente los desechos de la práctica clínica. Concluyendo que se realiza un adecuado un manejo de los residuos en general lo que reduce el riesgo de contagio del COVID-19. De la misma forma Herrera-Plasencia et al. (2020), menciona en su revisión de literatura científica en la profesión odontológica y el riesgo potencial de contaminación entre operados, asistentes y pacientes es alto. El odontólogo debe considerar a los pacientes como sospechosos de COVID-19, aplicando una adecuada gestión de bioseguridad mediante el uso de EPP, limpieza y desinfección del ambiente y superficies de contacto para reducir el riesgo de contagio.

\section{CONCLUSIÓN O CONSIDERACIONES FINALES}

Primera: Se concluye que entre la gestión de la bioseguridad odontológica y el riesgo de contagio por COVID- 19 del cirujano dentista de la comisión de salud bucal de Lima, 2021, existe una relación significativa y presenta una correlación positiva muy fuerte, $(\mathrm{p}=0,000$; rho de Spearman $=0,978)$, donde un 62,7\% afirma tener una adecuada gestión de la bioseguridad y un 61,8\% bajo riesgo de contagio COVID-19.

Segunda: Se concluye que entre la relación del cuidado personal odontológico y el riesgo de contagio por COVID-19 del cirujano dentista de la comisión de salud bucal de Lima, 2021, existe una relación significativa y una correlación positiva considerable, $(\mathrm{p}=0,000$; rho de Spearman $=0,851$ ), presentando el $69,1 \%$ un nivel alto de cuidado personal odontológico.

Tercera: Se concluye que entre la relación del manejo del material e instrumental y el riesgo de contagio por COVID-19 del cirujano dentista de la comisión de salud bucal de Lima, 2021, existe una correlación positiva media, $(\mathrm{p}=0,000$; rho de Spearman = 0,633), de la misma forma el $80 \%$ manifestó tener un nivel alto de manejo de material e instrumental.

Cuarta: Se concluye que entre la relación del ambiente odontológico y el riesgo de contagio por COVID-19 del cirujano dentista de la comisión de salud bucal de Lima, 
2021, existe una correlación positiva muy fuerte, $(\mathrm{p}=0,000$; rho de Spearman $=0,910)$ y que el 57,3\% reconoció tener un nivel alto del ambiente odontológico donde labora.

\section{LISTA DE REFERENCIAS}

Abreu, J. (2012). Método y Diseño de Investigación. International Journal of Good Conscience. 7(2), 187-197.

American Dental Association (2020). Develops guidance on dental emergency, nonemergency care. Disponible en: https://www.ada.org/en/publications/adanews/2020-archive/march/adadevelops-guidance-on-dental-emergency nonemergency-care

Alava-Zambrano, D., Ávila-Jalca, M., Castro-Jalca, J. (2021). Seroprevalencia de la COVID-19, factores de riesgo y protocolos de bioseguridad en el personal de salud de la ciudad de Jipijapa-provincia de Manabí- Ecuador. Polo del Conocimiento: Revista científico - profesional, 6(7), 3-18.DOI: 10.23857/PC. V6I7.2825.https://academic.microsoft.com/paper/3176205938/related

Almanza J., Calderón, C., y Vargas-Hernández, J. (2018). Teorías clásicas de las organizaciones y el Gung Ho. Revista Científica "Visión de Futuro", 22, (1). Disponible en: https://www.redalyc.org/articulo.oa?id=357959311001

Andersen, K., Rambaut, A., Lipkin, W., Holmes, E.y Garry, R. (2020). The proximal origin of SARS-CoV-2. Vigilancia epidemiologica aplicada proceso de enfermero.Nat.Med.;26(4):4502.pdf.Disponibleen:http://www.munayi.uleam.ed u.ec/wp

Aquino-Canchari, C. (2020). COVID-19 y su repercusión en la Odontología. Rev. cuba. estomatológica; $\quad$ 57(1): $\quad$ e3242, 2020. https://pesquisa.bvsalud.org/portal/resource/es/biblio-1126496

Arredondo, A. (1992) Analysis and Reflection on Theoretical Models of the HealthDisease Process. Cad. Saúde Públ., Rio de Janeiro, 8 (3): 254-261. Disponible en:https://www.scielo.br/j/csp/a/8bsQRMHDrQqWspcjLfhB8Qh/?format=pdf\&l ang=es

Arroyo, A. (2020). Metodología de la investigación en las ciencias empresariales [Tesis de Maestría, Universidad Nacional San Antonio de Abad del Cuzco]. http://repositorio. unsaac.edu.pe

Barabari P y Moharamzadeh K. (2020) Novel Coronavirus (COVID-19) and Dentistry-A 
Comprehensive Review of Literature. Dent J (Basel).21;8(2):53. DOI: 10.3390/dj8020053. PMID: 32455612; PMCID: PMC7345990.

Barbosa Liz, D., Agudelo Suárez, A., Atuesta Mondragón, MF. Ariza Olaya, J. y Plaza Ruíz, S. (2021) Dental practice modification, protocol compliance and risk perception of dentists during COVID-19 pandemic in Colombia: a cross-sectional study. Rev Fac Odontol Univ Antioq., 33(1), 17-35. DOI: http://dx.doi.org/10.17533/udea.rfo.v33n1a2

Benli, Merve; Huck, Olivier y Özcan, Mutlu. (2021). Awareness and precaution attitude of dentists as regards to risks associated with exposure to COVID-19 / Conscientização e atitude de precaução dos dentistas em relação aos riscos associados à exposição a COVID-19. Braz. dent. sci ; 24(4): 1-13.

Cagetti, M., Cairoli, J., Senna A. y Campus, G. (2020). COVID-19 Outbreak in North Italy: An Overview on Dentistry. A Questionnaire Survey. Int J Environ Res Public Health, 17(11),38-35.

Cari, H. y Zúñiga, E. (2017). Cumplimiento de las normas técnicas para el Manejo de residuos sólidos en una clínica universitaria,Juliaca. Evid En Odontol Clínica.3(7).

CIGET (2020). Un análisis de la percepción del riesgo ante la Covid-19. Ciencia en su PC, 1(2),1-24. Recuperado de https://www.redalyc.org/journal/1813/181363909001/html/

Cohen, C. y Shang, J. (2015) Evaluation of conceptual frameworks applicable to the study of isolation precautions effectiveness. J Adv Nurs.71(10),2279-92.

Coulthard, P. (2020) Dentistry and coronavirus (COVID-19) - moral decision making. Br. Dent. J., 228(7),503-5. DOI: https://www.doi.org/10.1038/s41415-020-14821

COVIDental Collaboration Group (2021). The COVID-19 pandemic and its global effects on dental practice. An International survey. Journal of Dentistry. Volume114.https://doi.org/10.1016/j.jdent.2021.103749.https://www.sciencedire ct.com/science/article/pii/S0300571221001706

Cuba, H. (2021). La pandemia en el Perú. Acciones, impactos y consecuencias del Covid19. Disponible en https://www.cmp.org.pe/wp-content/uploads/2021/05/LaPandemia-CUBA-corregida-vale.pdf 
Estrich, C. G., Mikkelsen, M., Morrissey, R., Geisinger, M. L., Ioannidou, E., Vujicic, M., y Araujo, M. W. B. (2020). Estimating COVID 19 prevalence and infection control practices among US dentists. The Journal of the American Dental Association, 151(11), 815-824. DOI: 10.1016/j.adaj.2020.09.005

Guerrero-Ramírez R, Meneses-La Riva ME, De La Cruz-Ruiz M. (2017). Cuidado humanizado de enfermería según la teoría de Jean Watson, servicio de medicina del Hospital Daniel Alcides Carrión. Lima- Callao, 2015. Rev. Enfermería Hered., $9(2), 133$.

Gómez Santos, G. y Prieto, R. (2021). Actualizando la práctica asistencial. Los cambios de la atención odontológica en atención primaria debido al SARS-CoV-2. FMC Formación Médica Continuada en Atención Primaria, 28(6), 318-323 https://doi.org/10.1016/j.fmc.2020.11.008

Hernández, R. y Mendoza, Ch. (2018). Metodología de la investigación: Las rutas cuantitativa, cualitativa y mixta. Ciudad de México, México: Editorial Mc Graw

Herrera-Plasencia, Paul, Enoki-Miñano, Erika; Ruiz-Barrueto, Miguel (2020). Riesgos, contaminación y prevención frente al COVID-19 en el quehacer odontológico: una revisión. Rev. salud pública, 22(5),300. https://pesquisa.bvsalud.org/portal/resource/es/biblio-1181001

Kraemer MUG, Yang CH, Gutierrez B, Wu CH, Klein B, Pigott DM; Open COVID-19 Data Working Group, du Plessis L, Faria NR, Li R, Hanage WP, Brownstein JS, Layan M, Vespignani A, Tian H, Dye C, Pybus OG y Scarpino SV. (2020). The effect of human mobility and control measures on the COVID-19 epidemic in China. Science, 368(6490):493-497. DOI: 10.1126/science. abb4218.

Kreps, G. (1996). La Comunicación en las Organizaciones. 2da ed. Estados Unidos: Editorial Addison-Wesley Iberoamericana.

Lai, T. H. T.; Tang, E. W. H.; Chau, S. K. Y.; Fung, K. S. C. y Li, K. K. W. (2020).

Mahase, E. (2020). Covid-19: WHO declares pandemic because of "alarming levels" of spread, severity, and inaction. doi: 10.1136/bmj.m1036

Marchena Pejerrey, Z. (2021). Gestión de la bioseguridad odontológica y riesgo de contagio por COVID-19 del cirujano dentista en el distrito de Chiclayo [Tesis de Maestría, Universidad César Vallejo]. Repositorio Institucional UCV. https://repositorio.ucv.edu.pe/handle/20.500.12692/57899 
Mendoza Murillo, P. y Mendoza Murillo, D. (2020). Riesgo de transmisión y bioseguridad del SARS-COV-2 por aerosoles generados durante los $\begin{array}{llll}\text { procedimientos } & \text { odontológicos. } & \text { Revistasunfv.8 }\end{array}$ 10.24039/CV202081764.

Meng, L., Hua, F. y Bian Z. (2020). Coronavirus Disease 2019 (COVID-19): Emerging and Future Challenges for Dental and Oral Medicine. J Dent Res, 99(5),481-487. Disponible: https://journals.sagepub.com/doi/full/10.1177/0022034520914246?url_ver=Z39. 88-2003\&rfr_id=ori:rid:crossref.org\&rfr_dat=cr_pub\%3dpubmed

Menjivar, I., Platero, C y Salinas, C. (2021). Verificación del protocolo de desinfección y esterilización del instrumental utilizado en clínicas dentales de unidades de salud del área Metropolitana De San Salvador Establecido Por El Ministerio De Salud en el Periodo de marzo-mayo de 2007 [Tesis de Doctorado, Universidad de El Salvador]. http://ri.ues.edu.sv/id/eprint/7946/1/17100308.pdf

Miguelena Muro, Karla Eugenia; García Esquivel, María Isis; López González, Lorena; Garcilazo Gómez, Alfredo; Tenorio Torres, Gustavo y Jiménez Días, Francisco David. (2021) Impacto de la pandemia en la práctica de los profesionistas de la salud oral en México / Impact of the COVID-19 pandemic in the oral health professionals' practice in Mexico. Rev. ADM; 78(3): 142-148, mayo-jun. 2021. ilus, graf

MINSA (2020). COVID 19 en el Perú. Recuperado el 03 de agosto de 2021. Disponible en: https://covid19.minsa.gob.pe/sala_situacional.asp

MINSA (2020). RM_456-2020-MINSA.pdf. Recuperado el 03 de agosto de 2021.

Disponible en: https://cdn.www.gob.pe/uploads/document/file/931760/RM_456-2020-

Naranjo-Hernández, Concepción Pacheco y Rodríguez Larreynaga (2017). La teoría Déficit de autocuidado: Dorothea Elizabeth Orem. Gaceta Médica Espirituana, 19(3), 89-100. Recuperado en 07 de agosto de 2021, de http://scielo.sld.cu/scielo.php?script=sci_arttext\&pid=S1608$89212017000300009 \& \operatorname{lng}=$ es \&tlng=es.

Ningthoujam, R. (2020). COVID 19 can spread through breathing, talking, study estimates. Curr. Med. Res. Pract. 10(3):132-3.

Organización Mundial de la Salud (OMS) (2020). Informe sobre Nuevo coronavirus - 
China. Disponible en: https://www.who.int/csr/don/12-january-2020novelcoronavirus-china/es/

Pérez, M. et al. (2020). Características clínico-epidemiológicas de la COVID-19. Rev. Habanera Ciencias Médicas. Disponible en: http://scielo.sld.cu/scielo.php?script=sci_abstract\&pid=S1729519X2020000200 005\&lng=es\&nrm=iso\&tlng=es

Putrino A, Raso M, Magazzino C, Galluccio G. (2020). Knowledge, management of patients and clinical experience of Italian dentists during the spread of contagion. BMC Oral Health Disponible https://www.ncbi.nlm.nih.gov/pmc/articles/PMC7349471/

Ramos, C. (2017). Los paradigmas de la investigación científica. Avances En Psicología. $23,9-17$.

Remuzzi, A. y Remuzzi, G. (2020). COVID-19 and Italy: what next? Lancet, 395(10231):1225-8.

Rocafuerte, M. (2020). La saliva y su papel en la trasmisión del Covid-19 - lo que el odontólogo necesita saber. KIRU. Revista de la Facultad de Odontología, 17(2). https://www.aulavirtualusmp.pe/ojs/index.php/Rev-Kiru0/article/view/1888

Sánchez, H., Reyes, C., Mejía, K. (2018). Manual de términos en investigación científica, tecnológica y humanística. Repositorio Institucional Universidad Ricardo Palma. http://repositorio.urp.edu.pe/handle/URP/1480

Segredo PAM.(2017). Instrumento para la evaluación del clima organizacional en salud. Revista Cubana de Salud Pública, 43(1),57-67.

Sigua-Rodríguez EA, Bernal-Pérez JL, Lanata-Flores AG, Sánchez-Romero C, Rodríguez-Chessa J, Haidar ZS, et al. (2020). COVID-19 y la Odontología: una Revisión de las Recomendaciones y Perspectivas para Latinoamérica. Int J. Odontostomatol,14(3), 299-309.

Universite Johns Hopkins (2020). COVID-19 Map - Johns Hopkins Coronavirus Resource Center. Recuperado el 04 de agosto de 2021. Disponible en: https://coronavirus.jhu.edu/map.html

Urquía, L. (2019). Teorías dominantes y alternativas en epidemiología. Edunla Cooperativa 2a ed. ISBN 978-987-4937-20-9. http://capacitasalud.com/wpcontent/uploads/2019/02/Teorias-dominantes-y-alternativas-de-la- 
epidemiologia.pdf

Vera (2020). El cirujano dentista y protocolo de bioseguridad frente al COVID 19.

Colegio odontólogos.16(1). https://www.cop.org.pe/uncategorized/decano-delcop-informo-que-cirujano-dentistas-seran-vacunados-con-dosis-de-refuerzoentre-el-9-y-13-de-noviembre

Wong, R., Kwong, T. Wu, J., Chan, M., Chu, S., Lee, H., Wong y Lung, D. (2020). Riesgo de transmisión nosocomial de la enfermedad por coronavirus 2019: una experiencia en un entorno de sala general en Hong Kong.J Hosp Infect, 105, pp. 119 - 127. DOI: 10.1016 / j. jhin.2020.03.036

Woodall, Irene R. et al. (1995). Tratado de Higiene Dental. Tomo II. España: Salvat.

World Health Organization (WHO). (2020). Novel Coronavirus - China. Recuperado el 04 de agosto de 2020. Disponible en: https://www.who.int/csr/don/12-january2020-novelcoronavirus-china/en/

Zambrano Guerrero, J. (2020). Letalidad en odontólogos diagnosticados con COVID-19, Perú 2020. [Tesis de Doctorado, Universidad Católica Santo]. 